\title{
SVM Applied to the Generation of Biometric Speech Key
}

\author{
L. Paola García-Perera, Carlos Mex-Perera, and Juan A. Nolazco-Flores \\ Computer Science Department, ITESM, Campus Monterrey \\ Av. Eugenio Garza Sada 2501 Sur, Col. Tecnológico \\ Monterrey, N.L., México, C.P. 64849 \\ \{paola.garcia, carlosmex, jnolazco\}@itesm.mx
}

\begin{abstract}
In this research we present a new scheme for the generation of a biometric key based on Automatic Speech Technology and Support Vector Machines. Keys are produced by making a distinction among the voice attributes of the users employing hyperplanes. It is described how the key is conformed and the reliability of the method. We depict an experimental evaluation for different values of the parameters. Among the different kernels for the Support Vector Machine, the RBF obtained the best results.
\end{abstract}

\section{Introduction}

Human body characteristics such as fingerprints, retinas and irises, facial structure, and voice recognition are just some of the many biometric fields being researched today. These characteristics are unique to each individual, then they are a good choice for developing biometric keys. The goal of the biometric key is to produce a password using the intrinsic attributes of some specific user characteristic with the consequence that remembering a complex password would not be necessary. Since voice is a natural process and it can produce different utterances in short time, it gives advantages among other biometrics. For instance, to assure user's identity the system can always request to him a random passphrase, avoiding the use of recorded voice by an unauthorised person. In this research we focus on voice and its characteristics to properly develop a reliable biometric key. This type of key generation has several applications in security purposes; for instance, biometric authentication and access to networks.

Through the years an extensive research has been done in the voice field. One of the branches is focused on the Automatic Speech Recognition (ASR), which deals with the computerised modelling of the human voice to obtain a transcript of what it is said. The previous research and advances done in the field together with the SVM techniques act as a basis for this investigation.

The main challenge of this research is to produce a biometric key that should repeatedly be equal for the same user utterance under certain conditions. Monrose et. al [6] showed a method to produce this key. The main point of their research was to map the voice features and providing a binary-classifying plane 
generate a descriptor from them. However, it had a serious drawback, the search of the partition plane was difficult due to the fact that infinite planes were possible. The key depended strictly on the plane chosen, this means that the key of the user was unknown until the plane was set. The number of trials to produce a suitable plane might be too large before obtaining the desired result. A more flexible way to produce a key is always attractive. Control of the assignation of the key values can enhance the cryptographic performance. It is also interesting to explore the possibility to produce a key by the use of an algorithm that can handle all the voice important attributes without discarding any of them.

In the next section we present our proposal. Section 3 through 5 describe the fundamental elements of the feature generation and SVM techniques. Section 6 examines the experimental methodology and the final section presents the results and discusses future research problems.

\section{Proposal}

The purpose of this proposal is to produce a biometric key from the attributes of the voice signal and the spoken user passphrase. The imperative challenge lies in the search of suitable set of planes that can significantly partition the handled data and give a key as a result.

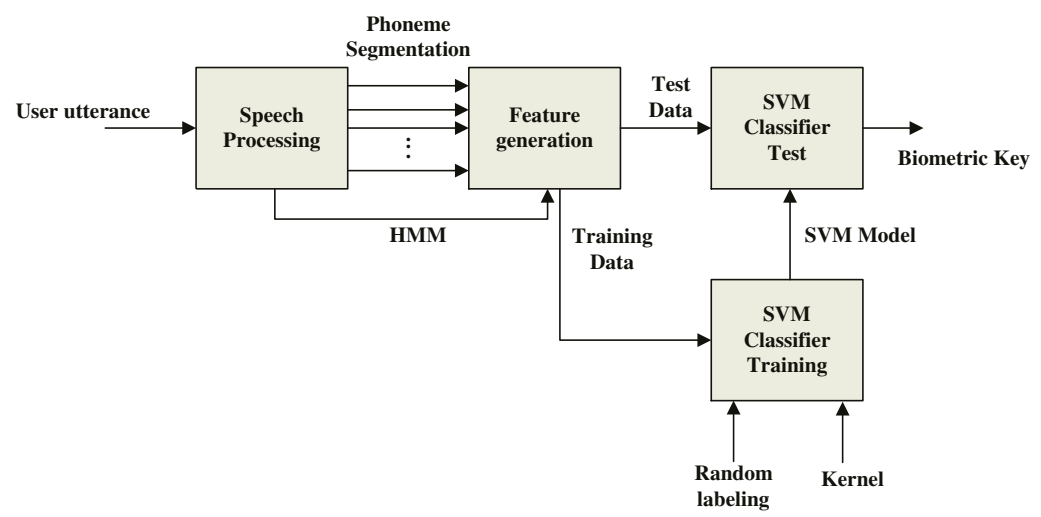

Fig. 1. System Architecure

A general view of the mechanism is shown in Figure 1. In the first stage, speech processing employing Automatic Speech Recognition techniques is used to extract the model parameters and the sets of phoneme frames of each user utterance. Manipulating both new sets of data the features are conformed. The Support Vector Machine classifier (SVM) will produce a new model according to the kernel and bit specifications. Finally, using the SVM model the key is generated. Each part will be discussed in the following sections. 


\section{Speech Processing}

The speech data is processed using Automatic Speech Recognition [11]. ASR provides two important results for the purpose of this research: an acoustic model named Hidden Markov Model (HMM) that has the inherent characteristics of real speech, and a set of phoneme-based segmented data. The HMM can be seen as a finite state machine, where output distributions are commonly represented by Gaussian Mixture Densities with means and covariances as important parameters.

Let $P$ be the set of phonemes, the states and the gaussians will make reference to this set. The phonemes were chosen as the sound units to be modelled since its possible to generate larger keys than working with the complete word. $C_{P}$ will denote the set of the central gaussian of the middle state vectors given by the HMM. This vector was chosen because the middle state is the most stable part of the phoneme representation.

\section{Feature Generation}

After obtaining the phoneme-based segmented data, the segment sets $\left\{R_{i, j}\right\}$ where $i$ is the index associated to each phoneme and $j$ is the $j$-th user, are conformed. Then, the feature vector is defined as $\psi_{i, j}=\mu\left(R_{i, j}\right)-C_{i}$, where $\mu\left(R_{i, j}\right)$ is the mean vector of the data in the $R_{i, j}$ segment, and $C_{i}$ is the matching phoneme mean vector of the model. Let us denote the universe set as $\Psi=\left\{\psi_{i, j} \mid\right.$ $1 \leq i \leq \ell, 1 \leq j \leq n\}$, where $\ell$ refers to the number of all the possible phonemes and $n$ is the number of all users.

Let $D_{p}$ be a subset of $\Psi$ containing all features related to a certain phoneme $p$. Thus, $D_{p}=\left\{\psi_{i, j} \mid i=p, 1 \leq j \leq n\right\}$. The final subsets $D_{p}$ are the input to the following step.

\section{Support Vector Machine}

This part of our research refers to the Support Vector Machine (SVM) technique used to produce a key. The SVM was chosen among others because a classifier that can separate sparse data and tolerate missclassification was needed.

The SVM is a method widely used for classification derived by Vapnik and Chervonenkis $[1,3]$. The goal of basic SVM is to obtain a model of vector classification in one of two classes. Two stages conform the SVM process: training and testing. Before the training process, the data should be formatted in vectors, and each vector is labelled according to its class. The following set of pairs are defined $\left\{x_{i}, y_{i}\right\}$; where $x_{i} \in R^{n}$ are the training vectors and $y_{i}=\{-1,1\}$ are the labels, see Figure 2.

For the data depicted in Figure 2 one can obtain a canonical form for $(w, b)$ of the unique separating hyperplane satisfying $y_{i}\left(w^{T} x_{i}+b\right) \geq 1$. The extension to the nonlinear technique consists on the nonlinear mapping of the input data into a high dimensional space. This transformation is denoted as function $\phi(x)$ and 


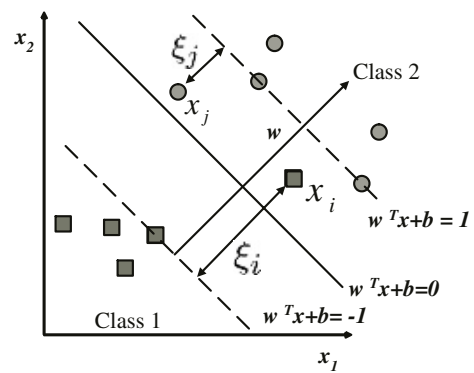

Fig. 2. Support Vector Machine

allows us to work in a huge dimensional space without doing explicit computation in this space. Then, the construction of the linear hyperplane is done. For the nonlinear case, $y_{i}\left[w^{T} \phi\left(x_{i}\right)+b\right] \geq 1$, and the optimisation problem is as follows,

$$
\begin{gathered}
\min _{x_{i}, b, \xi} \frac{1}{2} w^{T} w+C \sum_{i=1}^{l} \xi_{i} \\
\text { subject to } y_{i}\left(w^{T} \phi\left(x_{i}\right)+b\right) \geq 1-\xi_{i} \\
\xi_{i} \geq 0
\end{gathered}
$$

where $\xi_{i}$ is a slack variable and $C$ is a positive real constant known as a tradeoff parameter between error and margin.

It is possible to transform Equations 1 and 2 into a dual problem in the Lagrange multipliers $\alpha_{i}$. Thus,

$$
\begin{gathered}
\sum_{i=1}^{l} \alpha_{i}-\frac{1}{2} \sum_{i=1, j=1}^{l} \alpha_{i} \alpha_{j} y_{i} y_{j}\left\langle\phi\left(x_{i}\right), \phi\left(x_{j}\right)\right\rangle \\
\sum_{i=1}^{l} \alpha_{i} y_{i}=0, C \geq \alpha_{i} \geq 0 .
\end{gathered}
$$

The value of $\alpha_{i} \in R$ is close related to the training point $x_{i}$ and it represents the strength with which that point is associated in the final decision function, $\alpha_{i}$ can be solved as a quadratic programming (QP) problem. Only a subset of the points will be associated with a non-zero $\alpha_{i}$. These points are called support vectors and are the points that lie closest to the separating hyperplane.

The vectors are mapped into a higher dimensional space by function $\phi$. However exact specification of $\phi$ is not needed: instead, the expression known as kernel $K\left(x_{i}, x_{j}\right) \equiv \phi\left(x_{i}\right)^{T} \phi\left(x_{j}\right)$ is defined. There are different types of kernels, but the most common are

- linear $K\left(x_{i}, x_{j}\right)=x_{i}^{T} x_{j}$

- polynomial $K\left(x_{i}, x_{j}\right)=\left(\gamma x_{i}^{T} x_{j}+r\right)^{d}, \gamma>0$ 
- Radial Basis Function (RBF) $K\left(x_{i}, x_{j}\right)=e^{\left(-\gamma\left\|x_{i}-x_{j}\right\|^{2}\right)}, \gamma>0$

- Sigmoid function $K\left(x_{i}, x_{j}\right)=\tanh \left(\gamma x^{T} x_{j}+r\right)$

where $\gamma, r$ and $d$ are parameters.

The decision function is expressed as

$$
y(x)=\operatorname{sign}\left[\sum_{k=1}^{\# S V} \alpha_{k} y_{k} K\left(x_{i}, x\right)+b\right] .
$$

The parameters of the kernels should be tunned to find the best classification of the training data. When convergence is reached, the kernel and its final parameters are evaluated using Equation 5 and the test data. The statistics can give a guide on the performance of the classifier and decide to tune or stop.

The Support Vector Machine has been used for several applications, including biometrics $[10,9]$. In this research we employ it for searching a function that can be able to transform a vector produced by one phoneme to a binary number (key bit) assigned randomly.

The methodology used to implement the SVM training is as follows. Suppose that $/ A H /$ is the unique phoneme that can be uttered from all users. The one bit label $\{-1,1\}$ is assigned to each $D_{/ A H /}$. However, if one user utters the phoneme $/ A H /$ several times, the value of the bit will remain the same. For instance, $f\left(D_{/ A H /}\right)=1$ will be 1 for the utterances of the same user. The process is similar for the rest of the users, but the value of the bit $f\left(D_{/ A H /}\right)$ can change from user to user. The procedure is generalised for all the phonemes. Afterwards, the kernel parameters are tunned. In the test stage we evaluate the model produced by the SVM using unknown data. The statistics are made in terms of errors per phoneme according to different quantity of users.

As shown, the key is constructed using the phonemes of each word, and a random bit labeling. If the key has freedom of assignation the entropy grows significantly. This research considers just binary classes, however, an $M$-ary bit labeling per phoneme might be possible for future research. The final key could be obtained by concatenating the bits produced by each phoneme. For instance, if a user utters two phonemes: $/ F /$ and $/ A H /$, the final key is $K=$ $\left\{f\left(D_{/ F /}\right), f\left(D_{/ A H /}\right)\right\}$, thus, the output is formed by two bits.

\section{Experimental Methodology}

The experiments were performed using the YOHO database $[2,4]$. YOHO contains the voice utterances of 138 speakers of different nationalities speaking three pairs of numbers in each utterance. For instance, "Thirty-Two, Fourty-One, Twenty-Five".

The utterances are processed using the Hidden Markov Models Toolkit (HTK) by Cambridge University Engineering Department [5]. We employ HTK as an automatic speech recogniser. The important results of the speech processing stage are the twenty means of the phonemes given by the HMM and the phoneme-based segmentation of the utterances. The phonemes used are: $/ \mathrm{AH} /$, 
/AX/, /AY/, /EH/, /ER/, /EY/, /F/, /IH/, /IY/,/K/, /N/, /R/, /S/, /T/, $/ \mathrm{TH} /, / \mathrm{UW} /, / \mathrm{V} /, / \mathrm{W} /$. The model of each phoneme is composed by three states, and three gaussians per state. The mean of the central gaussian of the middle state of each phoneme and the phone-based segments of the utterances are the input for the feature generator. Following the method already described, the subsets $D_{p}$ are formed. It is important to note that the cardinality of each set $D_{p}$ can be different since the number of the same phoneme utterances can vary from user to user. Subsets from each $D_{p}$ were formed for training and test SVM phases. For training, the number of vectors picked for generating the model is the same. Each user has the same probability to produce the correct bit per phoneme. The number of test vectors that each user provided can be different.

Then, the assignation of $\{1,-1\}$ is performed. For simulation purposes, a distribution of equal number of 1's and -1's is given among the users. Then, the data is ready to be classified. SVMLight by Thorsten Joachims was used to implement the classifier [12]. We explored the performance of the linear, polynomial and RBF kernels. Suitable values of the parameters are choosen to produce the minimum amount of errors.

\section{$7 \quad$ Experimental Results}

One of the first stages in the experimental part was the selection of the parameters for the SVM. The behaviour of the SVM is given in terms of the average classification accuracy on test data for a given number of users. The average classification accuracy is computed by the ratio

$$
\eta=\frac{\text { matches on test data for all phonemes and users }}{\text { number of trials }} .
$$

In Figure 3 values for $\eta$ using the linear $(d=1)$, polynomial $(d>1)$, and $\operatorname{RBF}(\gamma=0.007)$ kernels are shown. It can be observed that the best results for the polynomial and linear case are obtained if the value of $d$ is equal to 2 or 3 . As long as $d$ increases, i.e. 5 , the accuracy of the SVM is reduced, classification is not performed for $d=7$. Moreover, analysing the RBF kernel, it can easily concluded that its behaviour is better than the linear or polynomial kernels although the increment of the number of users reduces the value of $\eta$.

The RBF kernel can model closed decision surfaces [8], which may occur in the speech case in view of the nature of the data. However a deeper research is suggested. Parameters $\gamma$ and $C$ must be adjusted in order to maximise $\eta$. From several experiments was concluded that a suitable value of $C$ is 6 . To see the behaviour of the SVM for different values of $\gamma$, some experiments were done. Figure 4 displays such results using $C=6$ for 10 users. If $\gamma$ decreases, it leads to a smoother decision surface; as $\gamma$ increases, the variance of the RBF decreases and produces a narrow support region (increment of the support vectors). The accuracy has its maximum values when $\gamma=0.007$ and $\gamma=0.009$.

As exposed the RBF kernel is a good solution in the generation of the key since it can give the maximum values for $\eta$, however an extra study should be done to produce the same effect when incrementing the users. 


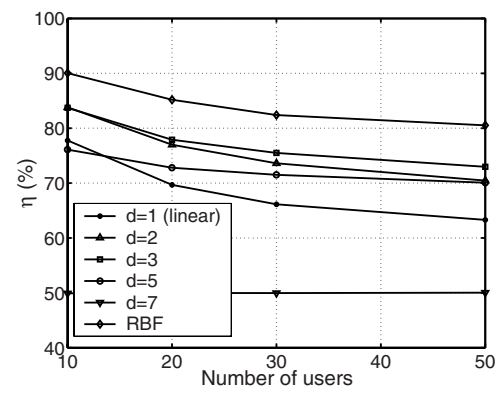

Fig. 3. $\eta$ for different number of users and several types of kernels

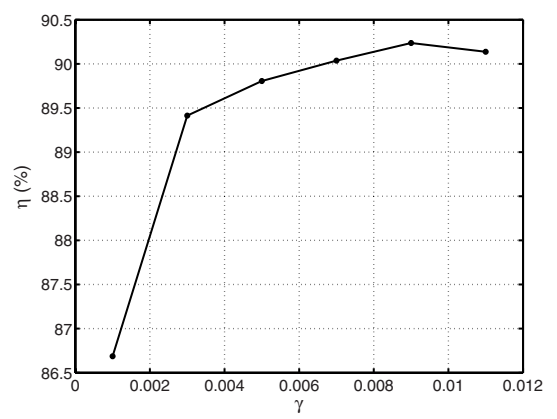

Fig. 4. $\eta$ for different values of $\gamma, C=6$, and 10 users

\section{Conclusion}

We have presented a method to produce a biometric key from voice based on a phoneme segmentation, where one key bit was assigned to each user phoneme. Combined techniques of ASR and Support Vector Machines have been used in this work. To our knowledge, Monrose et. al [6] has shown a first method to produce this key. The main advantage of our approach over Monrose work is that key bits can be selected and assigned for each user before the partition plane is computed. Thus, key bits are freely assigned regardless of the parameters value for the algorithms used in our method.

From the experiments conducted on several groups of users, it is concluded the feasibility of the use of the SVM to classify data vectors derived from users voice where the classes are labeled arbitrarily.

Among all the kernels used in the tests, RBF had the best performance, as discussed. The method to distinguish phonemes of specific users is quite good. However error correction mechanisms has to be considered in future research since the bits of the key must not present any error, although the number of users increment. Adding extra bits for error correction demands more phonemes in the passphrase but it might reduce the possibility of wrong key production.

Besides, future studies on a $M$-ary key can be useful to increase the number of different keys available for each user given a fixed number of phonemes in the passphrase. 


\section{Acknowledgments}

The authors would like to acknowledge the Cátedra de Seguridad, ITESM, Campus Monterrey and the CONACyT project CONACyT-2002-C01-41372 who partially supported this work.

\section{References}

1. Boser, B., I. Guyon, and V. Vapnik. A training algorithm for optimal margin classifiers. In Proceedings of the Fifth Annual Workshop on Computational Learning Theory, 1992.

2. Campbell, J. P., Jr. Features and Measures for Speaker Recognition. Ph.D. Dissertation, Oklahoma State University, 1992.

3. Cortes, C. and V. Vapnik. Support-vector network. Machine Learning 20, 273-297, 1995.

4. Higgins, A., J. Porter and L. Bahler. YOHO Speaker Authentication Final Report. ITT Defense Communications Division, 1989.

5. HTK Hidden Markov Model Toolkit home page. http://htk.eng.cam.ac.uk/

6. F. Monrose, M. K. Reiter, Q. Li , S. Wetzel. Cryptographic Key Generation From Voice. Proceedings of the IEEE Conference on Security and Privacy, Oakland, CA. May, 2001.

7. R. Morris and K. Thompson. Password security: A case history. Communications of the ACM, 22(11):594-597, 1979.

8. K.-R. Müller, S. Mika, G. Rätsch, K. Tsuda, and B. Schölkopf. An introduction to kernel-based learning algorithms. IEEE Neural Networks, 12(2):181-201, May 2001.

9. E. Osuna, R. Freund, and F. Girosi. Support vector machines: Training and applications. Technical Report AIM-1602, MIT A.I. Lab., 1996.

10. E. Osuna, R. Freund, and F. Girosi, Training Support Vector Machines: An Application to Face Recognition, in IEEE Conference on Computer Vision and Pattern Recognition, pp. 130-136, 1997.

11. L.R. Rabiner and B.-H. Juang. Fundamentals of speech recognition. Prentice-Hall, New-Jersey, 1993.

12. T. Joachims, SVMLight: Support Vector Machine, SVM-Light Support Vector Machine http://svmlight.joachims.org/, University of Dortmund, November 1999. 